\title{
Variations sur un thème unique : Les pratiques de la langue française de 1790 à 1960 au sein de l'Église Catholique Romaine en Louisiane.
}

\author{
Sylvie Dubois \\ Gabrielle Muir Professor in French Studies \\ Center for French and Francophones Studies \\ Louisiana State University \\ sdubois@1su.edu
}

\begin{abstract}
Durant les quinze dernières années, j'ai étudié, avec mon équipe, les changements intergénérationnels et les pratiques sociales des communautés francophones et anglophones dans le sud de la Louisiane. Et j'ai souvent éprouvé de la frustration face à l'absence de données empiriques sur les premiers changements de langue en Louisiane. Paradoxalement, c'est un événement dramatique qui a favorisé l'évolution de nos recherches : à la suite de l'ouragan Katrina, qui a inondé la Nouvelle-Orléans à la fin du mois d'août 2005, les documents religieux et cléricaux de la collection de l'Archevêché de la Nouvelle-Orléans ont été temporairement déplacés vers le Diocèse de Bâton-Rouge. Avec l'aide des archivistes du Diocèse, nous avons répertorié et étudié cette collection inexploitée de documents administratifs, de registres sacramentels et de lettres personnelles écrites entre 1803 et 1859 par la population locale, laïque et religieuse, des paroisses de Louisiane aux évêques de la Nouvelle-Orléans. L'accès à la correspondance de la période Antebellum (lettres personnelles écrites avant la Guerre civile), une des plus importantes en son genre en Amérique du Nord, nous a permis de recenser et d'analyser plus de 9000 lettres écrites en français et en anglais. C'est le résultat de cette recherche qui est présenté ici.
\end{abstract}

\section{Introduction}

Après un bref historique de l'Église catholique romaine en Louisiane, nous expliquerons pourquoi l'Archevêché de la Nouvelle-Orléans, en tant que base administrative de tout le territoire de la Louisiane, peut être défini comme un réseau institutionnel de communauté de pratiques. Nous résumerons ensuite les résultats de notre premier cas d'étude : le passage du français à l'anglais, en utilisant les données des registres sacramentels de plus de 173 Églises catholiques du sud de la Louisiane, de 1844 (le tout premier changement) à 1954 (le dernier changement). Toutefois, examiner le changement langagier dans les registres sacramentels ne se fait pas sans difficultés. Notamment, il n'y a pas toujours de césure claire et facilement explicable dans le passage du français à l'anglais dans la rédaction des registres; il y a aussi le problème d'un brusque changement langagier dans les nouveaux registres pré imprimés qui apparaissent dans certaines églises de la Louisiane au tournant du $20^{\mathrm{e}}$ siècle. Bien que certains changements dans les registres puissent être attribués à l'arrivée d'un nouveau prêtre anglophone, beaucoup d'autres sont initiés par des prêtres francophones déjà établis sur place depuis longtemps ou encore nouvellement arrivés. Les prêtres décident parfois de passer du français à l'anglais au début d'une nouvelle année, ou encore lorsqu'ils commencent un nouveau registre. Mais, dans de nombreux cas, il n'existe aucun indice émanant des registres susceptible expliquer ce changement de pratique.

Les registres ne permettant pas de résoudre toute une série d'interrogation, il était nécessaire de prendre en compte la structure hiérarchique d'autorité de l'Église catholique de la Louisiane, les contraintes sociales et les attitudes de ses membres, si nous voulions élucider non seulement la vitesse, mais également la source du changement langagier. Ainsi, nous nous sommes tournés vers d'autres collections d'archives qui nous ont 
permis de révèler l'existence de certaines contraintes et attitudes qui ne sont qu'implicites dans les registres. Nous avons examiné les documents administratifs, l'orgine des prêtres siègeant aux conseils, les rapports annuels des prêtres ainsi que les rapports sur le nombre et les pratiques langagières des paroissiens.

\section{L'établissement de l'église catholique romaine en louisiane.}

Pendant tout le $19^{\mathrm{e}}$ siècle et la première moitié du $20^{\mathrm{e}}$ siècle, l’Église catholique romaine a constitué le dernier vestige de la suprématie de la culture française et le dernier bastion du français écrit en Louisiane. Établi en 1793 et connu à l'origine comme le Diocèse de la Louisiane et des Florides, l'Archevêché de la NouvelleOrléans était une création commune du roi d'Espagne et du Pape. L'Église catholique louisianaise était composée d'une population multi-ethnique de fidèles, de prêtres séculiers et de prêtres réguliers c'est à dire appartenant à des ordres religieux. Cette population a préservé et nourri la foi catholique en construisant des églises, des paroisses, des écoles, des orphelinats, des hôpitaux et d'autres institutions sociales. De 1809 à 1860, le profil démographique de la population louisianaise a changé radicalement avec l'arrivée des protestants, qui se sont établis essentiellement dans le nord de la Louisiane et qui ont créé une division au sein de l'état. Seul le sud de la Louisiane est resté un territoire profondément catholique.

Tel que Dolan (1973:526) l'observe, une telle diversité ethnique a amené l'Église catholique louisianaise, comme partout ailleurs en Amérique du Nord, à adopter «le concept de la paroisse nationale ou de congrégation principalement organisée par la langue plutôt que par les limites du territoire ». De 1835 à 1860 , plus de 60 nouvelles églises de paroisses ont été établies, dont 20 pour la seule ville de la Nouvelle-Orléans. À l'exception du premier évêque pendant la période coloniale espagnole, tous les évêques et les archevêques étaient des francophones de naissance jusqu'à la nomination de l'archevêque allemand Francis Janssens en 1888. Les limites géographiques de l'Archevêché de la Nouvelle-Orléans entre 1853 et 1918 représentaient 35 paroisses civiles (comtés) dans le sud de la Louisiane. En 1900, l’Archevêché comptait une population de 325 000 catholiques (plus ou moins $25 \%$ de la population louisianaise), 161 églises et 211 prêtres pour servir le sud de la Louisiane. Aujourd'hui, la région est divisée en cinq diocèses, incluant le Diocèse de Baton-Rouge, Lafayette, Houma-Thibodaux, Lake Charles et l'Archevêché de la Nouvelle-Orléans.

\section{La représentation de l'église catholique louisianaise}

Selon Dulles (1978:39), l'Église catholique romaine se voit comme une société parfaite «qui n'est subordonnée à aucune autre et à laquelle il ne manque rien de nécessaire à sa propre perfection institutionnelle ». N'étant ni une démocratie, ni une instance publique représentative, l'Église catholique s'est cristallisée autour du concept d'autorité hiérarchique. Pour s'acquitter de ses tâches - c'est-à-dire enseigner, sanctifier et gouverner - l'Église catholique a prescrit des formes de cultes, a reconnu des ministres, des officiers responsables et a dûment défini les règles imposées aux fidèles. Une vue traditionnelle de l'Église catholique louisianaise ressemblerait à une structure pyramidale autoritaire avec, au sommet, l'administration de l'Archevêché avec un évêque - la liaison entre la « province de la Louisiane » et Rome - qui pourrait être comparé à un président et à un Président Directeur Général d'une société. L'administration de l'Archevêché contrôle et confie ses fonctions ecclésiastiques à des prêtres qui conseillent sur la conduite religieuse.

Cette représentation structurelle est problématique puisqu'elle implique une totale centralisation des pouvoirs, une unité dans les décisions et une application uniforme des règles (incluant les règles linguistiques), alors qu'en fait l'évolution linguistique qui a pris place dans cette institution - comme nous le démontrerons - n'a pas progressé de cette manière. Quelle autre forme de structure décisionnelle pourrait mieux expliquer les sources et la diffusion d'un changement de langue au sein de l'Église catholique louisianaise?

Afin de comprendre la complexité des contraintes qui régissent le changement de langue, nous avons choisi une approche différente. Nous préférons décrire l'Église catholique louisianaise comme un réseau de 
communauté de pratiques, un concept relativement nouveau dans le domaine de la sociolinguistique, préconisé par les spécialistes tels que Eckert et McConnell-Ginet (1992), Wenger (1998) et Meyerhoff (2002). Selon nous, la structure organisationnelle de l'Église catholique louisianaise incarne le concept de la communauté de pratiques: " un ensemble de personne qui se regroupe par engagement mutuel dans une entreprise » (Eckert \& McConnell-Ginet 1992 :464). Leur entreprise, en tant qu'institution, est la diffusion et le maintien de la foi catholique et de son enseignement au sein de la population locale.

Le noyau de l'Église catholique louisianaise du $19^{\text {e }}$ siècle était l'administration de l'Archevêché avec son Conseil Épiscopal. Cette communauté de pratiques (connue sous le nom de Curia) était composée d'hommes d'église haut placés et de prêtres locaux qui participaient à l'administration quotidienne' ${ }^{1}$. Entourant l'administration de l'Archevêché, on retrouvait une constellation de différentes communautés de pratiques représentées par les paroisses. Ces dernières étaient servies par des prêtres « séculiers » (aussi appelés prêtre de « diocèse ») et par des prêtres « réguliers» (en anglais religious) qui appartenaient à un ordre (tels les Jésuites, les Dominicains, les Joséphites, etc...). Plusieurs fidèles ou paroissiens étaient des membres importants de ces paroisses qui participaient aux activités administratives de la paroisse (la Fabrique ou conseil administratif, gestion de la propriété, écoles, etc...). La participation sociale de la plupart de ces membres peut être découverte à partir de toute une série de documents écrits (correspondance, rapports de visites de la paroisse, données provenant des registres sacramentels).

Les quatre dimensions de la communauté de pratiques de Wenger, c'est-à-dire la participation et la réification, la conception et l'émergence, le local et le global, et l'identification et la négociabilité, sont toutes facilement identifiables à l'intérieur de chacune des communautés de pratiques.

La dimension la plus significative dans le cadre de notre étude est probablement celle de l'identification et de la négociation. Elle nous permet d'identifier la relation complexe entre la communauté de pratiques de l'Archevêché et celles des paroisses et ainsi d'identifier la source et le type de pression sociale qui a créé le changement langagier. Il est important de noter que, malgré leur allégeance à l'Église catholique romaine, chaque communauté de pratiques forme une entreprise unique qui a sa propre vision et ses stratégies et qui entretient son propre réseau de membres. Les négociations à l'intérieur de ces entreprises (leurs conditions locales, le nombre et l'origine de leurs membres) et entre elles (qui travaille avec qui et contre qui) orchestre de façon ultime la pratique de leurs croyances. Il nous faut maintenant rechercher comment, à travers un engagement mutuel, ces communautés de pratiques ont négocié explicitement et implicitement le passage du français à l'anglais.

\section{Le changement langagier à l'intérieur des paroisses}

Les documents d'archives que nous avons sélectionnés pour notre premier cas d'étude sont les registres sacramentels qui enregistraient les baptêmes, les mariages et les enterrements d'individus pratiquants dans les paroisses. Ces moments importants de la religion catholique sont catalogués sous la forme de notes textuelles séparées plutôt que sous la forme de simples listes de noms. Habituellement, chaque note est écrite à la main et est signée par le prêtre et les témoins de l'événement. Selon le style d'écriture du prêtre, un registre peut couvrir 50 ans d'histoire locale, alors qu'un autre peut n'en couvrir que 10.

Le tableau 1 nous montre la moyenne des changements langagiers à travers le temps selon les diocèses et des paroisses. Il est clair que la plupart des changements dans les registres sont apparus au tournant du $20^{\mathrm{e}}$ siècle. Les églises, qui appartiennent aujourd'hui à l'Archevêché de la Nouvelle-Orléans (ou encore celles situées autour de la Nouvelle-Orléans) ont en moyenne procédé au changement une décennie plus tôt (1891) que celles du diocèse de Baton-Rouge (1906). Les églises du diocèse de Lafayette ont tenu leur registres en français jusqu'en 1917 et celles de Houma/Thibodaux ont changé en 1916, approximativement 26 ans après la Nouvelle-Orléans. La figure 2 est un histogramme qui représente le changement langagier au cours de cette 
période. Plusieurs paroisses sont passées du français à l'anglais à la fin du $19^{\mathrm{e}}$ siècle et au tournant du $20^{\mathrm{e}}$; toutefois, la majorité a changé entre 1900 et 1930. En fait, 46\% des églises de l'Archevêché ont opéré le changement entre 1880 et 1920 . La plupart des paroisses du sud de la Louisiane, qui ont changé après 1920, sont situées à l'ouest (Iberia et St-Martin) et au sud (Lafourche et St-James).

En fait, l'observation qui est probablement la plus importante à faire ici est que la moyenne des changement langagiers en Louisiane (1906) s'avère beaucoup plus tardive qu'on ne le dit généralement. En effet, la plupart des spécialistes qui ont décrit la situation de la langue en Louisiane au $19^{\mathrm{e}}$ siècle ont décrit le passage du français vers l'anglais comme un événement survenu soudainement à la fin de la Guerre civile, un peu comme l'abolition de l'esclavage. Il a souvent été affirmé ou suggéré que les francophones ont subitement arrêté d'écrire en français et qu'ils sont passés à l'anglais du jour au lendemain. Les données ici nous suggèrent tout autre chose. Certes, personne ne va nier qu'à la veille de la guerre civile, d'importants changements sociaux ont conditionné le choix de langue des prêtres locaux. Néanmoins, même si nous considérons la Reconstruction comme le catalyseur du monolinguisme anglophone, il n'en reste pas moins que le passage du français à l'anglais comme langue utilisée par la majorité des prêtres locaux a pris deux décennies de plus et pour certaines paroisses, le changement ne s'est pas effectué avant la deuxième Guerre Mondiale.

Examinons maintenant les changements langagiers par rapport à la situation géographique des églises. Avant l'achat de la Louisiane en 1803, il y avait 11 églises catholiques établies. La première église catholique répertoriée avec des registres en anglais est l'église irlandaise Saint Patrick de la Nouvelle-Orléans, construite en 1833 (Nolan 2000). Écrire les registres sacramentels en anglais a été manifestement une pratique introduite par les églises irlandaises de la Nouvelle-Orléans. Dès le début, tous leurs registres sacramentels ont été écrits en anglais. La seule exception est Sainte-Thérèse d'Avila, une autre église irlandaise, qui est passé du français à l'anglais quatre ans après son édification. Cette vague de nouvelles églises s'adaptait à la nouvelle importance démographique de la population celte en Louisiane. Par le seul poids de leur nombre, les Irlandais ont été les premiers opposants à la domination du catholicisme francophone. Le nombre de registres en anglais a par la suite augmenté en raison de l'apparition de nouvelles églises anglophones dans des villes récentes de la partie nord et ouest de la Louisiane catholique.

Entre 1857 et 1880, on assiste à une sorte de sursaut de la pratique du français dans les paroisses. Même si le nombre de nouvelles églises avec des registres en anglais (particulièrement dans la partie nord de la Louisiane catholique) a augmenté pendant la Guerre Civile et la période de la Reconstruction, un total de 29 nouvelles églises utilisent le français dans leurs registres sacramentels, ce qui constitue plus de la moitié des nouvelles églises anglophones. Sept églises sont passées du français à l'anglais, trois de ces dernières peu après leur fondation. La présence du français est particulièrement frappante et elle suggère, encore une fois, qu'une période importante de bilinguisme a existé et a été maintenue, même après le ralentissement de la migration des anglophones. Durant cette période, l'Église catholique de la Louisiane peut être réellement vue comme profondement bilingue. Cette constatation implique également que le déclin du français comme langue de prestige, ou langage de tous les jours, n'était pas chose faite ; le «triomphe» de l'anglais n'était donc pas prédéterminé mais constitue plutôt le résultat, du moins en partie, d'évènements sociolinguistiques qui ont eu lieu dans les décennies suivantes.

Le changement langagier dans les registres sacramentels atteignit son apogée à la fin du $19^{\mathrm{e}}$ siècle quand plus de 22 registres d'églises sont passés à l'anglais. Deux directions spatiales peuvent être observées: 1) il y a davantage d'églises à la Nouvelle-Orléans et dans ses environs qui ont adopté l'anglais et 2) un mouvement perceptible de registres anglophones est observé des paroisses du nord aux paroisses du sud. Les églises anglophones s'implantèrent également dans des localités à forte prédominance francophone, à l'invitation de l'archevêque Janssens en 1888. Pour la plupart, ces églises étaient régies par l'ordre catholique anglophone des Joséphites et desservaient spécifiquement la communauté noire du sud de la Louisiane (St-Augustine à New 
Roads dans la paroisse de Pointe Coupée et St-Benedict the Moor à Bertrandville dans la paroisse de l'Assomption).

Durant la période suivante (1902-1919), la diffusion géographique est encore plus accentuée; le changement autour de la Nouvelle-Orléans est maintenant terminé et les registres anglophones sont encore plus nombreux dans les paroisses du sud le long du fleuve que dans celles de l'ouest. Avant la Première Guerre Mondiale, 15 foyers de résistance ont cédé et ont ratifié le changement. Ce n'est que durant la Deuxième Guerre Mondiale que la pratique du français a été définitivement abandonnée par les sept églises francophones qui résistaient encore, une dans la paroisse de St-Martin, une dans la paroisse de Vermillon, une dans la paroisse de l'Assomption, deux dans la paroisse de St-James et deux à Lafourche.

\section{La source du changement}

Nous voudrions aborder brièvement ici la source du changement langagier dans les registres sacramentels. Dans la mesure où les "catholiques irlandais" représentent la population de catholiques non francophones la plus significative en Louisiane, nous pouvons les considérer comme la source principale du changement langagier. En d'autres termes, l'Église catholique louisianaise est le cadre où la formation d'une communauté anglophone catholique a eu lieu à la Nouvelle-Orléans, la communauté irlandaise étant le moteur du changement. En plus des preuves évidentes constituées par le nombre d'églises et la localisation des registres écrits en anglais, deux importants facteurs historiques émergent pour soutenir cette hypothèse.

Tout d'abord, et en dépit de leur histoire mouvementée avec la langue anglaise, les Irlandais ont rapidement et fermement établi l'anglais comme langue de pouvoir à la Nouvelle-Orléans. Bien qu'un certain nombre d'Irlandais aient certainement appris le français, langue de prestige et des affaires de la Nouvelle-Orléans au $19^{\mathrm{e}}$ siècle, ils auraient été probablement mécontents, en tant que catholiques dévoués, d'avoir à écouter les sermons uniquement en français (Niehaus 2004[1966]). En second lieu, les Irlandais sont venus à la NouvelleOrléans avec un héritage crucial qu'aucun groupe précédent d'immigrants ne possédait, y compris les Français : une tradition d'activisme politique incluant la défense du catholicisme. Ainsi, lorsqu'ils se sont installés en Louisiane, leur tradition politique était intacte et le catholicisme est devenu non seulement toléré, mais dominant. En combinant la religion et la politique ouvertement et librement, ils ont rapidement désiré leur propre Église où « Dieu parle en anglais » (Niehaus 2004 : 429). Les Irlandais catholiques avaient la capacité, la volonté et une population suffisante pour influencer l'Église catholique louisianaise, qui était une proie relativement facile puisque les francophones catholiques provenaient de cultures dans lesquelles le catholicisme allait de soi et n'avait jamais vraiment été défié (ou, en tout cas, pas dans les mêmes proportions qu'en Irlande).

L'influence des Irlandais sur l'Église catholique louisianaise a, de surcroît, une dimension originale qui mérite d'être soulignée. Les Irlandais ont changé l'Église catholique louisianaise de l'intérieur, sans affrontement significatif. Il n'y a pas eu de bataille pour les âmes des fidèles entre Irlandais et Français. Cependant, les Irlandais avaient leurs propres pratiques sociales et parlaient la langue du nouveau dirigeant (les Américains). Leurs aspirations étaient de créer une communauté séparée et ils ont réussi à le faire en introduisant une concurrence permanente avec les catholiques francophones. Ils ont ainsi changé l'Église catholique louisianaise parce qu'ils se voyaient comme des pratiquants plus fervents que les francophones qu'ils considéraient comme négligents, et, chose étonnante, les administrateurs de l'Eglise catholique louisinaise partageaient leur avis et se sont rangés de leur côté sur des questions importantes (Doorley 2001). Ainsi, l'adhésion à une même ferveur religieuse a triomphé des solidarités linguistiques. 


\section{L'influence de l'administration de l'archevêché}

Il va sans dire que changer les registres les plus importants de l'Église catholique louisianaise du français à l'anglais reflète un changement social important en Louisiane. Néanmoins, aucun édit linguistique n'a été émis par l'administration de l'Archevêché. Il n'y a aucune preuve dans la littérature de l'Église catholique louisianaise (les rapports internes et la correspondance entre les évêques et les prêtres locaux) qu'un décret, une décision ou même une directive à propos d'une préférence linguistique n'ait jamais été rédigé par l'ordre local avant ou après la Guerre Civile. Toutefois, durant les années de service des évêques Janssens, Chapelle et Blenk, y a-t-il eu des preuves écrites d'un changement du français à l'anglais à l'intérieur de l'Archevêché ? Pour répondre à cette question, nous avons consulté trois sources matérielles : (1) une base de données provenant des répertoires catholiques de 1880 à 1917 qui inclut le pays d'origine et la position de tous les administrateurs qui ont fait partie du Conseil Archiépiscopal ; (2) le compte-rendu du Conseil Archiépiscopal, un registre de comptabilité de 141 pages qui débute en 1858 et qui se termine en 1921 ; et (3) les lettres pastorales écrites au clergé et à la laïcité entre 1844 et 1913.

Examinons maintenant la composition du Conseil Archiépiscopal de 1898 à 1917 et la langue dans laquelle le compte-rendu du Conseil a été écrit. Nous pouvons noter que les officiers francophones de naissance étaient majoritaires durant le mandat de Janssens et Chapelle. Il est vrai que la composition a changé quand le Conseil s'est élargi à neuf membres avec des Italiens, des Belges, des Allemands et des marguilliers irlandais de naissance, mais le nombre d'officiers francophones de souche est toujours resté élevé. Les commentaires métalinguistiques du compte-rendu montrent que les rencontres qui ont eu lieu entre 1887 et 1913 se déroulaient toutes en français.

Un réel changement apparaît pendant le mandat de Blenk, plus précisément entre 1908 et 1910. Pour la première fois, le nombre d'officiers d'origine autre que francophone dépasse celui des francophones de naissance. En 1914, seulement deux officiers sur neuf étaient d'origine française et la composition multiethnique du Conseil a lentement conduit à l'utilisation de l'anglais comme seule langue commune pendant les réunions. Le Recteur Pierre Scotti, qui était le greffier, est passé du français à l'anglais au milieu de la réunion de juillet 1908, mais a continué d'écrire en français le compte-rendu du Conseil et en a fait de même pour les suivants. Cependant, quand le Père Vincent, un francophone d'origine, a remplacé le Recteur Scotti en 1913, le compte-rendu est définitivement passé à l'anglais.

L'analyse de la langue utilisée dans les lettres pastorales envoyées au clergé et aux paroissiens nous révèle que le statut du français à l'intérieur de l'administration de l'Archevêché a changé à travers le temps. Entre 1844 et 1883, c'est à dire lorsque Blanc, Odin et Perché étaient évêques, les lettres pastorales étaient écrites en français et en anglais. La version anglaise était envoyée aux paroisses composées de fidèles anglophones (Irlandais) ou non francophones (Italiens ou Allemands), alors que la version française était distribuée aux paroisses francophones.

Peut-être par souci d'économie, l'évêque Leray introduisit alors la pratique d'une lettre bilingue unique, les deux langues étant côte à côte sur la même page (le français en premier). Il est intéressant de constater que l'évêque Janssens, qui a été loué pour avoir diminué la dette de l'Archevêché, a repris la pratique d'écrire des lettres séparées. L'évêque Chapelle a réintroduit la lettre pastorale unique avec une légère différence: l'anglais plutôt que le français apparaît en premier. Pendant la guerre entre l'Espagne et les États-Unis, l'évêque Chapelle a été envoyé comme délégué à Cuba et à Puerto Rico par le Saint Siège, et comme Envoyé Extraordinaire aux Philippines. Pendant ses absences, le Père Administrateur Rouxel et le Vicaire-Général Laval ont abandonné le format côte à côte et ont publié une seule édition de lettres pastorales avec le texte écrit en anglais en premier, suivi de celui en français, une pratique perpétuée par Blenk lorsqu'il a été nommé évêque en 1906. 
L'introduction de registres pré imprimés, tout d'abord en français, ensuite en français et en anglais et finalement uniquement en anglais, a sans doute encouragé les prêtres au changement langagier. Néanmoins, plusieurs d'entre eux avaient déjà commencé à rédiger leurs registres en anglais avant l'introduction de formulaires standardisés. Le passage du bilinguisme au monolinguisme de langue anglaise à l'intérieur de l'Archevêché s'avère être une réaction a posteriori à trois changements sociaux : 1) l'augmentation de la population en milieu urbain; 2) un nombre décroissant de francophones demandant les services religieux dans leur langue; 3) le nombre croissant de prêtres uniquement anglophones.

\section{Les contraintes internes et externes au changement}

Bien que reconnus pour leur conservatisme, les officiers de l'Archevêché ont tout de même compris que les prêtres locaux étaient les plus proches des fidèles. Le choix de la langue dans les registres sacramentels (et les sermons) était un problème laissé entre les mains des pasteurs locaux qui avaient une meilleure connaissance que les administrateurs des besoins de la congrégation locale.

Le passage à l'anglais dans les registres a rarement été accompagné de commentaires. Pour plusieurs églises, il n'y a aucun indice dans ces derniers qui pourrait nous éclairer sur la raison du changement. Nous avons dénombré dix de ces changements brusques pour lesquels nous ne pouvons déterminer ce qui a engendré une telle décision individuelle. Nos résultats démontrent que les prêtres bilingues en tant qu'individus ont eu un impact important dans le changement langagier des registres puisque la moitié de ces changements coïncident avec un changement de prêtre. Bien que, dans certains cas, de nouveaux prêtres anglophones aient dorénavant décidé d'écrire les registres en anglais, ce sont les prêtres bilingues qui ont initié la plupart des changements. Dans plusieurs cas, des prêtres d'origine francophone commençaient à utiliser l'anglais dans des registres qui étaient tenus en français par des prêtres anglophones.

En outre, plusieurs prêtres ont attendu un moment de transition plus propice pour passer à l'anglais - un registre, un nouveau calendrier, la langue maternelle du nouveau praticien. Vingt-six changements coïncident avec un changement de format de registres, principalement pendant les deux premières décennies du $19^{\mathrm{e}}$ siècle. Avant l'arrivée des registres pré imprimés en anglais, toutes les informations écrites à la main dans les registres de plusieurs églises (Abbeville, Arnaudville, Breaux Bridge, New Iberia, St-Martinville et Ville Platte du diocèse de Lafayette) étaient écrites en français. Durant une très courte période, les prêtres ont complété en français les registres pré imprimés en anglais pour ensuite passer l'un après l'autre à l'anglais.

Les rapports de paroisse, envoyés à l'Archevêché par chaque église, nous procurent d'autres indications sur le changement langagier. Les premiers rapports, tel le questionnaire de 1885, étaient en français et en anglais et donnaient des informations sur le nombre de catholiques (blancs et personnes de couleurs), le nombre d'écoles et d'élèves et le nombre de sacrements. Il y avait aussi une case pour y inscrire la langue, mais il est impossible de déterminer si le document notait la langue des paroissiens, des prêtres ou celle des services. La plupart des rapports (9 sur 15) proviennent d'églises de la Nouvelle-Orléans et les annotations à propos de l'utilisation du français, de l'anglais, de l'italien et de l'allemand dans les églises étaient nombreuses. En 1912, les rapports de paroisse incluaient la nationalité des paroissiens catholiques et le nombre de ceux qui comprenaient l'anglais. Un total de 189 rapports de 1912, 1915, 1934, 1948 et 1954 a été trouvé pour 39 églises paroissiales.

Trois scénarios de changements émanent de ces données, chacun correspondant à un nombre équivalent d'églises dans six paroisses civiles. De façon intéressante, la localité ou le fait que le passage d'une langue à l'autre corresponde à un changement de prêtre ou de livre ne conditionne pas cette catégorisation. Dans le premier scénario, un changement se produit quand la plupart des paroissiens (de 80 à 100\%) comprennent l'anglais. Il y a une forte corrélation entre le nombre de personnes bilingues et la motivation du prêtre à procéder au changement. Le deuxième scénario est similaire au premier, mais la corrélation est moins forte 
puisque le changement s'effectue quand la moitié des paroissiens (plus ou moins 50\%) comprend l'anglais. Dans le troisième scénario, les prêtres basculent vers l'anglais alors qu'aucun ou très peu de paroissiens comprennent cette langue, c'est-à-dire quand les conditions locales ne justifient pas l'abandon du français.

L'analyse des registres nous permet d'avancer que les nouveaux prêtres étaient à l'aise dans les deux langues et que le changement s'est fait pour d'autres raisons que celles liées à la compétence linguistique. Les données indiquent notamment que d'importants changements locaux les ont motivés à passer du français à l'anglais. Un tel changement a été l'établissement d'institutions protestantes dans des paroisses autrefois dominées par la foi catholique. Est-ce que le nombre grandissant d'églises protestantes a déclenché le changement langagier à un niveau local ? Une plus grande proportion d'organisations protestantes en un seul endroit aurait-elle obligé les prêtres locaux à passer du français à l'anglais plus rapidement, non seulement pour attirer plus de paroissiens vers la foi catholique, mais aussi pour éviter de perdre un nombre sans cesse croissant d'anglophones catholiques d'origine française ?

En utilisant le Recensement américain de 1890, nous avons calculé la proportion d'églises Méthodistes, Baptistes du sud, Baptistes de couleurs et Évangéliques comparée au nombre d'églises catholiques dans chacune des paroisses. Il y a une corrélation significative entre le nombre proportionnel d'églises protestantes et la date de passage du français à l'anglais. Autrement dit, plus la proportion d'églises protestantes est grande, plus tôt se fait le changement. Inversement, nous pouvons observer que les pratiques bilingues dans plusieurs paroisses, où le nombre d'églises protestantes et le niveau de compétition était relativement bas, ont été conservées jusqu'à la Deuxième Guerre Mondiale. Ce résultat nous suggère que le besoin de défendre la foi catholique en utilisant la langue dominante dans la localité a triomphé sur le conservatisme des prêtres locaux, même les pasteurs d'origine française.

\section{Conclusion}

Cette recherche fournit donc une preuve supplémentaire que la Louisiane a connu une période de bilinguisme au $19^{\mathrm{e}}$ siècle, et que le stéréotype selon lequel l'anglais a «chassé» le français a besoin d'être considérablement nuancé.

Pendant plus d'un siècle, l'Église catholique louisianaise a adopté une politique non-officielle de bilinguisme illustrée par des rapports administratifs écrits en français et en anglais. Ce laissez-faire et cette politique linguistique accommodante à l'intérieur de l'administration de l'Archevêché contrastent considérablement avec ce qui est advenu au niveau du gouvernement de l'État, où la politique de «l'anglais seulement» a été déterminée par décrets (cf. la Constitution de l’État de la Louisiane, 1868 et 1921).

La source du changement langagier à l'intérieur de l'Église catholique louisianaise est à rechercher dans la migration massive de catholiques irlandais, qui ont défié la tradition louisianaise de la messe en français. Ils ont aussi exigé des registres sacramentels écrit en anglais plutôt qu'en français ou en latin. En passant plus tard à l'anglais et en nommant des prêtres d'une origine autre que francophone à des positions clés dans les diocèses, l'administration de l'Archevêché a accentué un changement déjà en place dans les paroisses, un changement fortement lié à plusieurs évènements socio-historiques. Le sort du bilinguisme a été scellé par des contraintes socio-géographiques, sociales et attitudinales à leur apogée au tournant du 20e siècle, notamment en ce qui concerne le changement majeur dans les centres urbains au début de la période anticatholique. Deux contraintes importantes ont eu une influence critique sur la diffusion spatiale du changement langagier dans les registres sacramentels des paroisses. Pour beaucoup de prêtres, la décision de passer à l'anglais dans les registres a été provoquée par un changement interne dans leur église ; c'est-à-dire quand le nombre croissant de paroissiens anglophones et de paroissiens francophones comprenant l'anglais a justifié le changement. En d'autres termes, certains prêtres sont passé à l'anglais quand l'utilisation du français est devenue un obstacle à la propagation de la foi catholique. D'autres prêtres ont été influencées par un facteur externe : l'augmentation 
d'églises protestantes dans leur paroisse, particulièrement l'expansion d'organisations protestantes que rejoignaient tant les Blancs que les communautés noires pauvres. Cette pression a incité certains prêtres à passer à l'anglais malgré le nombre dominant de fidèles francophones.

À ce stade de notre réflexion, nous croyons avoir dévoilé un important fragment du casse-tête linguistique que pose la Louisiane du $19^{\mathrm{e}}$ siècle. Bien sûr, les documents d'églises ne représentent pas une image complète de la situation linguistique en Louisiane et nous savons que la problématique de la langue parlée était bien différente à plusieurs niveaux. Néanmoins, la forte corrélation entre le nombre d'églises protestantes et le changement linguistique dans les paroisses les plus importantes ajoute un autre fait statistiquement vérifiable à notre connaissance sur la longue et ultime guerre d'usure que se livrent, à ce moment essentiel de l'histoire, les deux pratiques linguistiques.

\section{Références bibliographiques}

Dolan, J. (1973) A critical period in American Catholicism. The Review of Politics 35:523-36.

Doorley, M. (2001). Irish Catholics and French Creoles: Ethnic struggles within the Catholic church in New Orleans, 1835-1920. The Catholic Historical Review 87a:34-54.

Dulles, A. (1978) Models of the Church. Garden City, NY : Doubleday.

Eckert, P. et S. McConnell-Ginet. (1992) Think practically and look locally: Language and gender as community-based practice. Annual review of anthropology 21:461-90.

Meyerhoff, M. 2002. Communities of practice. In Handbook of language variation and change, ed. J.K. Chambers, Peter Trudgill \& Natalie Schilling-Estes, 526-48. Oxford: Blackwell.

Niehaus, E. (2004[1966]) The Irish and their Church, 1830-1862. In The Louisiana Purchase bicentennial series in Louisiana, vol. 19. Religion in Louisiana, ed. Charles E. Nolan, 429-441. Lafayette la: Center for Louisiana Studies, University of Louisiana at Lafayette. (Reprinted from The Irish in New Orleans, 1966, 98111, 181-83. Baton Rouge: Louisiana State University Press.)

Nolan, C. (2000) A History of the Archdiocese of New Orleans. France : Éditions du Signe.

The Official Catholic Directory. (2004) New Providence nj: P.J. Kennedy \& Sons.

United States Census. (1894) Religious bodies. Report on statistics of churches in the United States at the 11th Census, 1890, by Henry K. Carroll, Special Agent, vol. 9. http://www.archives.gov.

Wenger, E. 1998. Communities of practice : Learning, meaning, and identity. Cambridge: Cambridge University Press. 


\begin{tabular}{|c|c|c|c|}
\hline \multicolumn{2}{|c|}{ Échelle géographique } & \multicolumn{2}{|c|}{ Date moyenne du changement } \\
\hline \multicolumn{2}{|l|}{ Louisiane (86) } & \multicolumn{2}{|l|}{1907} \\
\hline \multicolumn{2}{|c|}{ Archevêché Nouvelle Orléans (23) } & \multicolumn{2}{|l|}{1891} \\
\hline \multicolumn{2}{|c|}{ Diocèse Baton Rouge (25) } & \multicolumn{2}{|l|}{1906} \\
\hline \multicolumn{2}{|l|}{ Diocèse Lafayette (26) } & \multicolumn{2}{|l|}{1917} \\
\hline \multicolumn{2}{|c|}{ Diocèse Houma/Thibodeaux (12) } & \multicolumn{2}{|l|}{1916} \\
\hline Paroisses & Date changement & Paroisses & Date changement \\
\hline East Baton Rouge & une église 1854 & $\begin{array}{l}\text { St. John the Baptist } \\
\text { (3) }\end{array}$ & 1910 \\
\hline Orleans (9) & 1880 & Terrebonne (4) & 1910 \\
\hline Jefferson (2) & 1885 & St Landry (4) & 1911 \\
\hline Iberville (5) & 1887 & Lafayette (3) & 1905 \\
\hline Ascension (2) & 1887 & Acadia (5) & 1916 \\
\hline Pointe Coupée (3) & 1890 & Assumption (6) & 1919 \\
\hline St. Tammany (3) & 1893 & Vermilion (5) & 1921 \\
\hline St. Charles & une église 1898 & Iberia (3) & 1923 \\
\hline St. Bernard & une église 1899 & St. Martin (3) & 1924 \\
\hline Plaquemine (4) & 1900 & Lafourche (7) & 1926 \\
\hline $\begin{array}{l}\text { West Baton Rouge } \\
\text { (2) }\end{array}$ & 1901 & St. James (3) & 1926 \\
\hline St. Mary (4) & 1906 & Livingston & une église 1934 \\
\hline
\end{tabular}

Tableau 1. Date du changement linguistique dans les registres sacramentels en Louisiane, par diocèses et paroisses. Les nombres en parenthèses représentent le nombre d'églises analysées. 


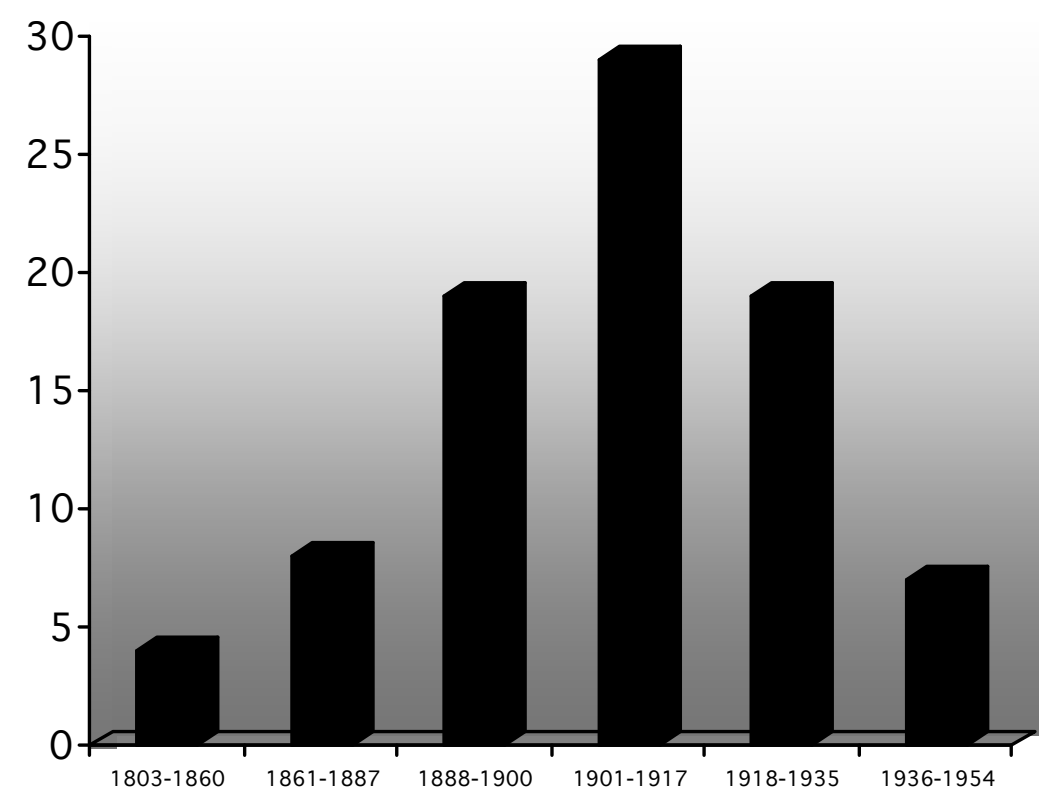

Figure 1. Changements langagiers de 1803 à 1954 dans les registres sacramentels par nombre de paroisses.

${ }^{1}$ L'archevêque représente l'Église catholique louisianaise, mais n'a aucune juridiction sur le diocèse. Les évêques agissent en tant qu'officiers exécutifs de l'Église catholique louisianaise et chacun dirige un diocèse. Ils sont entourés du cœur des membres de l'administration supérieure. 